\title{
Spectacles utilization and its impact on health-related quality of life among rural Chinese adolescents
}

\author{
Deng-Juan Qian ${ }^{1} \cdot$ Hua Zhong ${ }^{2} \cdot J_{u n} \mathrm{Li}^{3} \cdot \mathrm{Hu} \mathrm{Liu}^{4} \cdot$ Chen-Wei Pan ${ }^{1}$
}

Received: 5 August 2017 / Revised: 9 March 2018 / Accepted: 17 July 2018 / Published online: 29 August 2018

(c) The Royal College of Ophthalmologists 2018

\begin{abstract}
Purpose The aims of the study were to assess the magnitude and predictors of the unmet need for spectacles utilization and to quantify its impact on health-related quality of life (HRQOL) among rural Chinese adolescents.

Methods This school-based survey of 2346 grade- 7 students (mean age: 13.8 years) was conducted in Southwestern China in 2016. Spectacles need was defined as uncorrected visual acuity (VA) of 20/40 or worse but correctable to 20/40 or better in the better-seeing eye, together with the presence of myopia of less than -0.5 diopters (D), hyperopia of more than $+2.0 \mathrm{D}$, or astigmatism of more than $0.75 \mathrm{D}$ in both eyes. The HRQOL was measured using self-reported versions of 23-item Pediatric Quality of Life Inventory Version 4.0 Generic Core Scales (PedsQL 4.0).

Results Among 579 (24.7\%) adolescents with an uncorrected VA of 20/40 or less, 483 (83.4\%) needed vision corrections and $172(35.6 \%)$ used them. Higher parental education levels (odds ratio $(\mathrm{OR})=2.73 ; 95 \% \mathrm{CI}, 1.29-5.77)$, negative attitude regarding spectacles $(\mathrm{OR}=0.49 ; 95 \% \mathrm{CI}, 0.25-0.97)$, and poorer uncorrected $\mathrm{VA}(\mathrm{OR}=31.27 ; 95 \% \mathrm{CI}, 3.76-260.23)$ were independent determinants for spectacles utilization. Adolescents not using spectacles had a lower HRQOL score compared to those using spectacles in terms of psychosocial health $(65.91$ vs. $70.59 ; P=0.028)$, emotional health $(56.85$ vs. 63.24 ; $P=0.012)$, and social functioning (72.99 vs. $78.60 ; P=0.036)$.

Conclusions The rate of spectacles utilization in rural China was low. Adolescents were not accustomed to use spectacles had a worse HRQOL score.
\end{abstract}

\section{Introduction}

Vision problem is a global health concern, especially in children and adolescents [1,2]. China is the world's most

These authors contributed equally: Deng-Juan Qian and Hua Zhong.

Electronic supplementary material The online version of this article (https://doi.org/10.1038/s41433-018-0197-x) contains supplementary material, which is available to authorized users.

Chen-Wei Pan

pcwonly@gmail.com

1 School of Public Health, Medical College of Soochow University, Suzhou, China

2 Department of Ophthalmology, the First Affiliated Hospital of Kunming Medical University, Kunming, China

3 Department of Ophthalmology, the Second People's Hospital of Yunnan Province, Kunming, China

4 Department of Ophthalmology, the First Affiliated Hospital with Nanjing Medical University, Nanjing, China populous country and may have the greatest burden of vision loss throughout the world. According to a conservative estimate, there are more than 6 million children and adolescents aged from 5 to 15 years suffering from visual disabilities in China [1]. Uncorrected refractive errors contribute largely to vision loss in children and adolescents. The global prevalence of reduced vision due to uncorrected refractive errors ranges from 1.3 to $22.3 \%$ [3-5], with the highest rate being observed in Chinese adolescents [4]. Impaired vision, if untreated, poses significant socioeconomic burdens [2] and may lead to lifelong adverse consequences, such as reduced cognitive abilities [6], unstable emotional developments [7], poor academic performances [8], higher social unemployment rates [9], and impaired health-related quality of life (HRQOL) [10].

Spectacle is a cost-effective and safe intervention for refractive corrections $[8,11]$. Previous findings have indicated that the unmet need for refractive corrections is widespread [4, 12-14]. In China, for example, only 16-35\% of the students needing spectacles are actually wearing them $[15,16]$. However, few data are available in rural 
communities, where vision-related health resources are relatively limited. More importantly, although previous studies have reported that childhood vision disorders were related to a worse HRQOL [17-20], the impact of the unmet need of spectacles wearing on HRQOL remains unrevealed. Considering that HRQOL measurements are becoming increasingly important in clinical and health service research [21], it is imperative to quantitatively assess the impact of vision corrections on children's HRQOL, which are important to guide clinical managements and health resource allocations.

The Andersen Behavioral Model is a widely used sociological model for detecting the determinants of health services utilization in medical settings [21]. The model includes three structural components: predisposing factors, enabling factors, and need characteristics. As a mature theoretical framework, it has been widely used both in original investigations [22] and systematic reviews [23] regarding different health outcomes. To help with the explanation of school-based vision service utilization, the Andersen Behavioral Model [24] was employed to assess the predictors of spectacles utilization in this study.

The objectives of this study were (1) to describe the prevalence and determinants of spectacles utilization among rural Chinese adolescents by employing the Andersen Behavioral Model; and (2) to further examine the impact of the unmet need of spectacles utilization on generic HRQOL scores in the same cohort.

\section{Materials and methods}

\section{Participants}

This school-based survey was conducted in 2016 aiming at longitudinally observing the onset and progression of common vision disorders among school students in Mojiang, which is located in Southwestern China. Cohort participants were grade-7 students in middle schools who would be followed up annually until entering high schools. The compulsory schooling system in China would largely facilitate the establishment of the cohort and increase the follow-up rate. Based on the official records, there were totally of 2510 grade 7 students from ten middle schools in Mojiang. Ultimately, 2346 (93.5\%) of them with a mean age of 13.82 (standard deviation (SD): 0.84) years completed eye examinations in the study, including 1213 $(51.7 \%)$ boys and $1133(48.3 \%)$ girls. Permission was obtained from at least one parent or legal guardian of each participant by telephone calls and, if unavailable, through home visits.

Ethical approval was prospectively granted by the Institutional Review Board of Kunming Medical University.
Declaration of Helsinki involving human participants and the approved guidelines were followed throughout.

\section{Ocular examinations and procedure}

Extensive ocular examinations including visual acuity (VA) measurements, ocular motility evaluations, investigation of eye diseases by utilizing slit lamp and ophthalmoscopy, measurements of intraocular pressure by non-contact tonometer, tests for cycloplegia, post-cycloplegic refractions, measurements of ocular biometry data employing an IOL Master (Carl Zeiss Meditec AG, Jena, Germany) and the assessments of the external eye, anterior segment, media, and retinal fundus were carried out by optometrists or trained technicians at each school site.

Baseline VA test was performed separately on each eye with the right eye being tested first. In a well-lighted room with the illumination being 500 lux, study participants underwent a reduced retroilluminated logarithm of the minimum angle of resolution (logMAR) E-chart (Precision Vision, Villa Park, IL) at 4-m distance, starting from the top line of the chart, letter by letter. If students correctly read 4 of 5 letters or more on the 20/200 rows, they were asked to identify the 20/100 rows, the 20/50 rows, and the 20/10 rows, successively. The measurement of the previous line was recorded as the result if one incorrectly identified more than 1 letter in the row. Study participants who failed to read any character at the 4-m point, were ask to advance to the $1-\mathrm{m}$ point, progressing down the chart as described earlier and the result was divided by 4 [16, 25]. Presenting visual acuity (PVA) was defined as the number of letters read correctly without spectacles or with their habitual corrections on the day of the examination.

Two drops of cyclopentolate (1\%) were administered to each participant twice with $5 \mathrm{~min}$ apart. After $30 \mathrm{~min}$, if the pupil size was $<6 \mathrm{~mm}$ or the pupil was reactive, a third drop was applied. Afterwards, cycloplegic refraction was measured using an autorefractor (RM-8000; Topcon Corp., Tokyo, Japan). The average reading of five consecutive measurements of refraction were calculated for data analyses. All five readings should be at most 0.50 diopters (D) apart in both the spherical and cylinder components.

\section{Questionnaires}

Information regarding demographic characteristics, spectacles utilizations, myopia-related lifestyle exposures, details of ophthalmic histories, family wealth, parental histories of myopia, and parental educations was collected using predesigned questionnaires. The attitudes of participants towards spectacles utilization and eye care were collected. For example, agreement of "spectacles help improving performance for myopic students" was considered as 
positive attitude and disagreement was considered as negative. Body mass index (BMI, $\mathrm{kg} / \mathrm{m}^{2}$ ) was calculated by one's weight in kilograms divided by the square of one's height in meters. Furthermore, a Chinese version of the Children's Sleep Habits Questionnaire (CSHQ) [26] was also used to measure the presence of disordered sleep in this study.

\section{Instruments for measuring HRQOL}

We measured the HRQOL using the 23-item Pediatric Quality of Life Inventory Version 4.0 Generic Core Scales (PedsQL 4.0) $[20,21]$ as reported by each participant. The PedsQL 4.0 is a simple, well-developed and extensively used instrument for measuring HRQOL among adolescents aged 13 to 18 years. The instrument comprised of physical health (eight items), emotional health (five items), social functioning (five items), and school functioning (five items). Responses were made on a 5-point Likert scale with 0 denoting "never a problem" and 5 denoting "almost always a problem". Responses were reversely transformed to a 0 to 100 scale with higher scores representing a better HRQOL.

\section{Definitions of spectacles need and utilization}

The definition of spectacles need and utilization was similar to a previous study in Chinese children and adolescents [16]. Spectacles need was defined as either uncorrected VA of 20/40 or worse and could be improved with correction to be 20/40 or more in the better-seeing eye, together with the presence of myopia of $-0.5 \mathrm{D}$ or less, hyperopia of $+2.0 \mathrm{D}$ or more, or astigmatism of $0.75 \mathrm{D}$ or more in both eyes. Refractive errors were estimated based on the spherical equivalent refraction (spherical power $+1 / 2 \times$ cylindrical power). Spectacles utilization was defined based on wearing spectacles at school.

\section{Data analysis}

For the analysis of rates and determinants of spectacles utilization, adolescents without spectacles need were excluded $(n=1863)$. Multivariate logistic regression models were fitted to determine the odds ratios (ORs) and corresponding 95\% confidence intervals (CIs) of associated factors for spectacles utilization. Determinants of spectacles utilization were further categorized into predisposing, enabling and need factors based on the Andersen Behavioral Model. PedsQL scale values were estimated by adding the value of the 23 items and then dividing the sum by the number of items responded. All data were analyzed using SPSS V16.0 (Statistical Package for Social Science).
A two-sided $P<0.05$ was considered to be statistical significant.

\section{Results}

In this study, one in four adolescents had an uncorrected VA of 20/40 or less among the all the 2346 adolescents. Compared with adolescents with reduced VA (VA of 20/40 or worse), those with an uncorrected VA of better than 20/ 40 tended to be boys $(55.9 \%$ vs. $44.1 \% ; P<0.001)$, and have more hyperopic refraction $(0.31 \mathrm{D}$ vs. $-2.05 \mathrm{D} ; P<$ $0.001)$. Girls $(31.2 \%)$ were significantly more likely to be affected by vision problems than boys $(18.6 \%)(P<0.001)$. The prevalence of spectacles utilization was $35.2 \%(67 / 185)$ for girls and $36.2 \%(105 / 298)$ for boys with no significant sex difference $(P=0.45)$ among the 483 adolescents needing spectacles. The prevalence of reduced PVA (PVA of 20/40 or worse) was 17.4 and $82.6 \%$ among students using and not using spectacles, respectively $(P<0.001)$. The mean spherical equivalents of the two groups were $-3.18 \mathrm{D}$ and $-0.04 \mathrm{D}(P<0.001)$.

Compared with adolescents not using spectacles, those who used were taller, were more likely to be the only child in the family $(42.4 \%$ vs. $23.8 \% ; P<0.001)$, had better educated parents (having at least one parent with high school education or above, 47.7 vs. $22.2 \% ; P<0.001$ ), had higher family incomes (family income per month $>5000$ yuan, $22.1 \%$ vs. $9.0 \% ; P<0.001)$, lived in a larger house (family building area $>100 \mathrm{~m}^{2}, 33.7 \%$ vs. $21.2 \% ; P=$ 0.002 ), were more likely to report to have myopia by themselves $(96.5 \%$ vs. $83.0 \% ; P<0.001)$, held positive attitude towards spectacles (spectacles help improving performance for myopes, $87.5 \%$ vs. $79.0 \% ; P=0.031$ ), and had more myopic parents (having at least one myopic parent, $15.5 \%$ vs. $5.8 \% ; P=0.001)$. In contrast, they were less likely to hold negative attitude (spectacles make vision worse, $56.0 \%$ vs. $67.8 \% ; P=0.034$ ). There were no differences in terms of age, sex, parental attitudes towards spectacles wearing between the two groups (all $P>0.05$ ). (Table 1).

Consistent with the Andersen's behavioral model, demographics, attitudes towards spectacles utilization, and family structures were classified as predisposing factors. Enabling factors included family income and house area size in our study. Measured vision status and self-evaluated health status were fitted in need factors. Multiple logistic regression analysis revealed that parental education levels were positively associated with spectacles utilization (OR $=2.73 ; 95 \% \mathrm{CI}, 1.29-5.77)$. In turn, those who held the attitudes that spectacles made the vision worse were less likely to use spectacles $(\mathrm{OR}=0.49 ; 95 \% \mathrm{CI}, 0.25-0.97)$. 
Table 1 Characteristics of adolescents use or did not use spectacles

\begin{tabular}{|c|c|c|c|}
\hline Variables & $\begin{array}{l}\text { Use } \\
\text { spectacles } \\
(n=172)\end{array}$ & $\begin{array}{l}\text { Did not use } \\
\text { spectacles } \\
(n=311)\end{array}$ & $P$ \\
\hline \multicolumn{4}{|l|}{ Predisposing factors } \\
\hline Age, years & $13.7(0.8)$ & $13.8(0.8)$ & 0.57 \\
\hline Girls & $105(61.0)$ & $193(62.1)$ & 0.83 \\
\hline Height, cm & $155.2(7.3)$ & 152.4(7.9) & $<0.001$ \\
\hline$\geq 1$ Parent was myopic, $\%$ & $26(15.5)$ & $18(5.8)$ & 0.001 \\
\hline $\begin{array}{l}\geq 1 \text { Parent with education } \\
\text { of high school or above, } \%\end{array}$ & $82(47.7)$ & $69(22.2)$ & $<0.001$ \\
\hline Only child in family, $\%$ & $73(42.4)$ & $74(23.8)$ & $<0.001$ \\
\hline \multicolumn{4}{|c|}{ Parental attitudes towards wearing spectacles, $\%$} \\
\hline Support & $68(39.8)$ & 11(36.7) & 0.77 \\
\hline Do not care & $46(26.9)$ & $7(23.3)$ & \\
\hline Oppose & $57(33.3)$ & $12(40.0)$ & \\
\hline $\begin{array}{l}\text { Adolescents held the } \\
\text { attitude that spectacles } \\
\text { make the visual acuity } \\
\text { worse, \% }\end{array}$ & $56(56.0)$ & $120(67.8)$ & 0.034 \\
\hline $\begin{array}{l}\text { Adolescents holding the } \\
\text { attitude that spectacles } \\
\text { help improving } \\
\text { performance for myopes, } \\
\%\end{array}$ & $112(87.5)$ & $169(79.0)$ & $\mathbf{0 . 0 3 1}$ \\
\hline \multicolumn{4}{|l|}{ Enabling factors } \\
\hline \multicolumn{4}{|c|}{ Family income per month, yuan } \\
\hline$\leq 5000$ & 134(77.9) & 283(91.0) & $<0.001$ \\
\hline$>5000$ & $38(22.1)$ & $28(9.0)$ & \\
\hline \multicolumn{4}{|l|}{ Housing areas, $\mathrm{m}^{2}$} \\
\hline$\leq 100$ & $114(66.3)$ & $245(78.8)$ & 0.002 \\
\hline$>100$ & $58(33.7)$ & $66(21.2)$ & \\
\hline \multicolumn{4}{|l|}{ Need factors } \\
\hline $\begin{array}{l}\text { Uncorrected visual acuity } \\
\text { of }<20 / 40 \text { in both eyes, } \%\end{array}$ & $28(16.3)$ & $2(0.6)$ & $<0.001$ \\
\hline Self-reported myopia, \% & $166(96.5)$ & $258(83.0)$ & $<0.001$ \\
\hline
\end{tabular}

Data presented are means (SDs) or $n(\%)$, as appropriate for variable. Bold value indicates statistical significance $(\mathrm{p}<0.05)$.

For need factors, we found that the prevalence of spectacles utilization substantially increased with a poorer uncorrected VA $(\mathrm{OR}=31.27$; 95\% CI, 3.76-260.23). There was a nonsignificant trend towards a positive relationship between spectacles utilization and self-reported myopia $(\mathrm{OR}=1.92$; 95\% CI, 0.61-6.06). (Table 2).

The means and $95 \%$ CIs of the PedsQL sub-scale and total scale values stratified by the presence of spectacles utilization among adolescents needing them are shown in Table 3 . We observed marginally positive relationships between presence of spectacles and emotional health, social functioning and school functioning scale scores in univariate analysis (all $P$ values were between 0.05 and 0.10 ). After controlling for possible confounders such as age, sex, parental education, BMI, family income, number of children in the family, and the CSHQ values, those using spectacles still had significantly higher psychosocial health scores than those who did not use $(P=0.028)$. However, the PedsQL total scores and physical health scores did not show significant variations between the two groups after multivariate adjustments $(P>0.05)$.

\section{Discussion}

Our school-based investigation provided novel data on the magnitude and predictors of the unmet need of spectacles among adolescents in rural China. In general, spectacles utilization rate in rural China was similar to that of adolescents of similar ages in urban areas [16]. Those using spectacles had a lower HRQOL compared to those who did not use, particularly in terms of psychosocial health scores.

To the best of our knowledge, there has been a paucity of data focusing on spectacles utilization among adolescents in rural China. Our prevalence estimates of spectacles utilization among rural Chinese adolescents are comparable to those reported among similar-aged individuals in urban China using similar study protocols and definitions of the outcome variables [16]. In our study, the crude prevalence of spectacles utilization (35.6\%) was higher than that reported in rural-to-urban migrant students $(23.0 \%)$ but was similar to that among urban students (35.3\%) [16]. According to the Chinese national census data in the year of 2010 (http://www.stats.gov.cn/), there are around 8.6 million rural and 3.4 million urban adolescents aged 13 years in China. Based on the results of the current study, the projected numbers of rural adolescents who need spectacles were about 7.2 million, among whom, 4.6 million had not worn spectacles yet. This estimate was almost three times than their counterparts in urban communities in China. Thus, health practitioners and policy makers should be aware that more efforts and resources should be channeled towards addressing this major public health concern in rural communities in China.

In this study, parental education, adolescents' attitudes towards spectacles utilization and uncorrected VA were significant determinants to spectacles utilization after adjustment for potential confounders. As reported previously [27], low attention and consciousness of the need for visual corrections from parents and adolescents may lead to the low rates of spectacles utilization. Our results showed that adolescents who hold the attitudes that "spectacles make the vision worse" were less likely to wear spectacles. The finding highlights the necessity of the wide-ranged health education programs and supports the assertions of Andersen's behavioral model of health care utilization that individual factors affect health care 
Table 2 Multiple logistic regression models of the associated factors for spectacles utilization among adolescents

\begin{tabular}{lll}
\hline Variables & OR $(95 \% \mathrm{CI})$ & $\mathrm{P}$ \\
\hline Predisposing factors & & \\
Girls & $0.67(0.35,1.28)$ & 0.67 \\
$\geq 1$ Parent was myopic & $1.77(0.59,5.30)$ & 0.31 \\
$\geq 1$ Parent with education of high school or above & $2.73(1.29,5.77)$ & $\mathbf{0 . 0 0 8}$ \\
Only child in family & $1.85(0.83,3.82)$ & 0.10 \\
Adolescents holding the attitude that spectacles make the visual acuity worse & $0.49(0.25,0.97)$ & $\mathbf{0 . 0 4 1}$ \\
Adolescents holding the attitude that spectacles help improving performance & $1.04(0.44,2.47)$ & 0.93 \\
for myopic students & & \\
Enabling factors & & 0.29 \\
Family income per month, yuan & 1.00 & \\
$\leq 5000$ & $1.65(0.66,4.10)$ & \\
$>5000$ & & 0.001 \\
Need factors & $31.27(3.76,260.23)$ & $\mathbf{0 . 0 6}$ \\
Uncorrected visual acuity <6/12 in both eyes & $1.92(0.61,6.06)$ & 0.26 \\
Self-reported myopia &
\end{tabular}

CI confidence interval, $O R$ odds ratio utilization. Additionally, adolescents whose parents had a higher education level and a higher rate of spectacle wearing were more likely to wear spectacles. However, parental spectacle wearing was a predisposing factor of adolescents' spectacle utilization in univariate but not multivariate analysis (Table 3). Thus, parental spectacles wearing may be simply an indicator rather than an independent risk factor for spectacles utilization in offspring. Reduced VA has emerged as a strong indicator of need as suggested by other studies, given the blurred vision conditions [16].

Other than the predisposing factors of demographic factors in the original model, our work indicated that parental education is also a pertinent factor for spectacles utilization. The Andersen's model also incorporates predictors at the socio-political level of the medical resource [24] which is not collected in our case. Unquestionably, vision health services usage is a complex behavior because of the interplay of a myriad of factors. Future research should aim to elucidate some other factors in relation to spectacles utilization, such as need for care and health alternative resources.

Adolescents who did not use spectacles had statistically though not clinically worse emotional and social functioning as measured by the PedsQL after controlling for possible confounding variables. Numerous studies have explored the relationship between HRQOL and various ophthalmic conditions, such as glaucoma [17], strabismus [18], uncorrected refractive errors [7], and reduced VAs $[19,20]$. Reduction in HRQOL scores caused by eye disorders was substantial. For example, the Multi-ethnic Pediatric Eye Disease Study (MEPEDS) showed that strabismus was significantly linked with a worse HRQOL among preschool children [18]. Another example was a snapshot qualitative research, where lower vision-related quality of life was identified to be related to uncorrected refractive errors and refractive corrections were proven to be associated with subjective improvements, such as improved academic performance among Indian children [7]. Despite disparities in study protocols, our findings were consistent with the general conclusions of previous studies, which showed a negative impact of reduced VA or uncorrected VA on individual's HRQOL [7, 19, 20]. In addition to the impacts on collective activities, social life and thus lower social functioning, our study also suggested a deleterious impact of worse vision on emotional health of adolescents who did not use spectacles. The reasons for this phenomenon are visualized. Children or adolescents, especially girls, with vision problems are easy to be embarrassed, feel inferior and develop introvert personalities $[7,20]$. Nevertheless, moderate reduced VA or uncorrected refractive errors are always called "vision conditions" or "ocular disorders" rather than "eye diseases" and may influence slightly on adolescents compared with "eye diseases" such as cataract and glaucoma. Furthermore, our instruments for measuring HRQOL were generic rather than vision-specific and may not be sensitive enough to detect a remarkable reduction in the overall HRQOL scores between adolescents using or not using spectacles. Besides the studies on children and adolescents, the European Eye study [28] has drawn similar conclusions among adult populations, suggesting that the reduced VA caused by unmet need for spectacles may last from childhood to older life stage.

Considering that Chinese rural populations account for over $80 \%$ of the national population and have only limited access to health care services, understanding the prevalence, predictors, and impacts of the unmet need for spectacles in 
Table 3 Health-related qualityof-life values among adolescents use or did not use spectacles

\begin{tabular}{llll}
\hline Levels & Use spectacles $(n=172)$ & Did not use spectacles $(n=311)$ & $P$ \\
\hline Physical health & & & \\
Age and sex adjusted & $72.85(70.47-75.23)$ & $71.48(69.69-73.27)$ & 0.36 \\
Multivariate adjusted & $73.29(67.68-78.89)$ & $75.68(69.62-81.73)$ & 0.41 \\
Psychosocial health & & & $\mathbf{0 . 0 3 7}$ \\
Age and sex adjusted & $71.59(69.19-73.99)$ & $68.45(66.65-70.25)$ & $\mathbf{0 . 0 2 8}$ \\
Multivariate adjusted & $70.59(66.46-74.72)$ & $65.91(61.45-70.38)$ & 0.08 \\
Emotional health & & & $\mathbf{0 . 0 1 2}$ \\
Age and sex adjusted & $66.82(63.89-69.76)$ & $63.67(61.47-65.88)$ & \\
Multivariate adjusted & $63.24(58.28-86.20)$ & $56.85(51.49-62.21)$ & 0.09 \\
Social functioning & & & $\mathbf{0 . 0 3 6}$ \\
Age and sex adjusted & $77.53(74.57-80.48)$ & $74.46(72.24-76.67)$ & \\
Multivariate adjusted & $78.60(73.42-83.77)$ & $72.99(67.40-78.59)$ & 0.09 \\
School functioning & & & 0.60 \\
Age and sex adjusted & $70.18(67.45-72.92)$ & $67.24(65.18-69.29)$ & 0.36 \\
Multivariate adjusted & $69.64(64.74-74.54)$ & $68.32(63.02-73.62)$ & 0.36 \\
Total & & & $71.48(69.69-73.27)$ \\
Age and sex adjusted & $72.85(70.47-75.23)$ & $69.49(64.94-74.04)$ & \\
Multivariate adjusted & $71.48(67.27-75.69)$ & &
\end{tabular}

Data are presented as means value and $95 \%$ confidence intervals

Adjusted for age, sex, parental education, body mass index, family income per month, only child in the family, and Children's Sleep Habits Questionnaire values rural areas of China is of utmost importance from a public health perspective. Our findings revealed that visually impaired adolescents without spectacles had a significantly decreased psychosocial health score, which may result in mental health problems. Nowadays, reduction of health inequalities has become a main policy target in many countries and there is a universal recognition of the disadvantage of mental health $[29,30]$. Thus, it is essential for Chinese governments and health policy makers to plan affirmative-action programs related to vision health among rural school students.

To the best of our knowledge, the current study was the first one that employed the Andersen behavioral model to assess the determinants of spectacles utilization in rural China. We also had a large sample size, a high response rate and detailed clinical examinations. Our study was limited by the lack of information on societal determinants of eye care utilization, which are important composition of the original Andersen's behavioral model. Furthermore, in this study, we only assessed whether adolescents used spectacles but did not evaluate whether the spectacles they used were adequate. Last but not least, the cross-sectional design was limited to determine causation when analyzing risk factors.

In conclusion, a low rate of spectacle utilization was observed among Chinese adolescents living in rural communities. Our study also suggested that visually impaired adolescents without refractive corrections might have lower psychosocial health scores and subsequently a higher risk of mental health problems. These findings may have important public health implications for China and other resourcelimited economies with regards to the reduction of unmet need for spectacles among school-aged adolescents.

\section{Summary}

\section{What was known before}

- Childhood vision disorders were related to a worse health-related quality of life.

\section{What this study adds}

- Our work provided novel data on the magnitude, predictors, and impacts of the unmet need for spectacles utilization.

- Adolescents without spectacles had a worse healthrelated quality-of-life score.

Acknowledgements This work was supported by the National Natural Science Foundation of China under grant no. 81773449 and 81560169. The sponsor or funding organization had no role in the design or conduct of this research. 


\section{Compliance with ethical standards}

Conflict of interest The authors declare that they have no conflict of interest.

\section{References}

1. Resnikoff S, Pascolini D, Mariotti SP, Pokharel GP. Global magnitude of visual impairment caused by uncorrected refractive errors in 2004. Bull World Health Organ. 2008;86:63-70.

2. Gordois A, Cutler H, Pezzullo L, Gordon K, Cruess A, Winyard $\mathrm{S}$, et al. An estimation of the worldwide economic and health burden of visual impairment. Glob Public Health. 2012;7:465-81. https://doi.org/10.1080/17441692.2011.634815

3. Robaei D, Rose K, Ojaimi E, Kifley A, Huynh S, Mitchell P. Visual acuity and the causes of visual loss in a population-based sample of 6-year-old Australian children. Ophthalmology. 2005;112:1275-82. https://doi.org/10.1016/j.ophtha.2005.01.052

4. He M, Huang W, Zheng Y, Huang L, Ellwein LB. Refractive error and visual impairment in urban children in southern china. Invest Ophthalmol Vis Sci. 2004;45:793-9.

5. McCarty CA. Uncorrected refractive error. $\mathrm{Br} \mathrm{J}$ Ophthalmol. 2006;90:521-2.

6. Roch-Levecq AC, Brody BL, Thomas RG, Brown SI. Ametropia, preschoolers' cognitive abilities, and effects of spectacle correction. Arch Ophthalmol. 2008;126:252-8. https://doi.org/10. 1001/archophthalmol.2007.36. quiz 161

7. Kumaran SE, Balasubramaniam SM, Kumar DS, Ramani KK. Refractive error and vision-related quality of life in South Indian children. Optom Vision Sci. 2015;92:272-8. https://doi.org/10. 1097/OPX.0000000000000494

8. Ma X, Zhou Z, Yi H, Pang X, Shi Y, Chen Q, et al. Effect of providing free glasses on children's educational outcomes in China: cluster randomized controlled trial. BMJ. 2014;349:g5740 https://doi.org/10.1136/bmj.g5740

9. Rahi JS, Cable N, British Childhood Visual Impairment Study, G. Severe visual impairment and blindness in children in the UK. Lancet. 2003;362:1359-65. https://doi.org/10.1016/S0140-6736 (03)14631-4

10. Varma R, Wu J, Chong K, Azen SP, Hays RD; Los Angeles Latino Eye Study Group. Impact of severity and bilaterality of visual impairment on health-related quality of life. Ophthalmology. 2006;113:1846-53. https://doi.org/10.1016/j.ophtha.2006.04. 028

11. Li L, Song Y, Liu X, Lu B, Choi K, Lam DS, et al. Spectacle acceptance among secondary school students in rural China: the Xichang Pediatric Refractive Error Study (X-PRES)-report 5. Invest Ophthalmol Vis Sci. 2008;49:2895-902.

12. He M, Huang W, Zheng Y, Huang L, Ellwein LB. Refractive error and visual impairment in school children in rural southern China. Ophthalmology. 2007;114:374-82. https://doi.org/10.1016/j. ophtha.2006.08.020

13. Paudel P, Ramson P, Naduvilath T, Wilson D, Phuong HT, Ho $\mathrm{SM}$, et al. Prevalence of vision impairment and refractive error in school children in Ba Ria - Vung Tau province, Vietnam. Clin Exp Ophthalmol. 2014;42:217-26. https://doi.org/10.1111/ceo.12273

14. Mehari ZA, Yimer AW. Prevalence of refractive errors among schoolchildren in rural central Ethiopia. Clin Exp Optom. 2013;96:65-9. https://doi.org/10.1111/j.1444-0938.2012.00762.x

15. He J, Lu L, Zou H, He X, Li Q, Wang W, et al. Prevalence and causes of visual impairment and rate of wearing spectacles in schools for children of migrant workers in Shanghai, China. BMC Public Health. 2014;14:1312 https://doi.org/10.1186/1471-245814-1312

16. Wang X, Yi H, Lu L, Zhang L, Ma X, Jin L, et al. Population prevalence of need for spectacles and spectacle ownership among urban migrant children in Eastern China. JAMA Ophthalmol. 2015;133:1399-406. https://doi.org/10.1001/jamaophthalmol. 2015.3513

17. Freedman BL, Jones SK, Lin A, Stinnett SS, Muir KW. Visionrelated quality of life in children with glaucoma. J AAPOS. 2014;18:95-8. https://doi.org/10.1016/j.jaapos.2013.09.010

18. Wen G, McKean-Cowdin R, Varma R, Tarczy-Hornoch K, Cotter SA, Borchert M, et al. General health-related quality of life in preschool children with strabismus or amblyopia. Ophthalmology. 2011;118:574-80. https://doi.org/10.1016/j.ophtha.2010.06.039

19. Chadha RK, Subramanian A. The effect of visual impairment on quality of life of children aged 3-16 years. Br J Ophthalmol. 2011;95:642-5. https://doi.org/10.1136/bjo.2010.182386

20. Wong HB, Machin D, Tan SB, Wong TY, Saw SM. Visual impairment and its impact on health-related quality of life in adolescents. Am J Ophthalmol. 2009;147:505-11 e501. https:// doi.org/10.1016/j.ajo.2008.09.025

21. Varni JW, Seid M, Kurtin PS. PedsQL 4.0: reliability and validity of the Pediatric Quality of Life Inventory version 4.0 generic core scales in healthy and patient populations. Med Care. 2001;39:800-12.

22. Hochhausen L, Le HN, Perry DF. Community-based mental health service utilization among low-income Latina immigrants. Community Ment Health J. 2011;47:14-23. https://doi.org/10. 1007/s10597-009-9253-0

23. Babitsch B, Gohl D, von Lengerke T. Re-revisiting Andersen's behavioral model of health services use: a systematic review of studies from 1998-2011. Psychosoc Med. 2012;9:Doc11. https:// doi.org/10.3205/psm000089.

24. Andersen RM. Revisiting the behavioral model and access to medical care: does it matter? J Health Soc Behav. 1995;36:1-10.

25. Yi H, Zhang H, Ma X, Zhang L, Wang X, Jin L, et al. Impact of free glasses and a teacher incentive on children's use of eyeglasses: a cluster-randomized controlled trial. Am J Ophthalmol. 2015;160:889-96.e881. https://doi.org/10.1016/j.ajo.2015.08.006

26. Silva FG, Silva CR, Braga LB, Neto AS. Portuguese Children's Sleep Habits Questionnaire - validation and cross-cultural comparison. J Pediatr. 2014;90:78-84. https://doi.org/10.1016/j.jped. 2013.06.009

27. Odedra N, Wedner SH, Shigongo ZS, Nyalali K, Gilbert C. Barriers to spectacle use in Tanzanian secondary school students. Ophthalmic Epidemiol. 2008;15:410-7. https://doi.org/10. 1080/09286580802399094

28. Seland JH, Vingerling JR, Augood CA, Bentham G, Chakravarthy $\mathrm{U}$, deJong PT, et al. Visual impairment and quality of life in the older European population, the EUREYE study. Acta Ophthalmol. 2011;89:608-13. https://doi.org/10.1111/j.1755-3768. 2009.01794.x

29. Daponte A, Bernal M, Bolívar J, Mateo I, Salmi LR, Barsanti S, et al. Criteria for implementing interventions to reduce health inequalities in primary care settings in European regions. Eur $\mathbf{J}$ Public Health. 2014;24:980-90. https://doi.org/10.1093/eurpub/ cku044

30. Mangalore R, Knapp M, Jenkins R. Income-related inequality in mental health in Britain: the concentration index approach. Psychol Med. 2007;37:1037-45. https://doi.org/10.1017/ S003329170600969X 